М.Ю. Петранова ${ }^{1}$

Моделювання гауссового стаціонарного процесу зі стійкою кореляційною функцією із заданою надійністю та точністю

1 Донецький національний університет імені Василя Стуса, 21021, м. Вінниця, вул. 600річчя, 21

e-mail: m.petranova@donnu.edu.ua
M. Yu. Petranova ${ }^{1}$

\section{Simulation of a Gaussian stationary process with a stable correlation function with a given reliability and accuracy}

${ }^{1}$ Vasyl' Stus Donetsk National University, 21021, 600-richchia str., 21

e-mail: m.petranova@donnu.edu.ua

У иій статті для побудови моделей стохастичних процесів, що наближае процеси із заданою надійністю та точністю в просторі $L_{p}([0, T])$ використано зображення випадкових процесів у вигляді випадкових рядів з некорельованими членами, отримані в роботі Ю. В. Козаченко, I. В. Розора, Є. В. Турчина (2007) [1]. Подібні конструкчії були досліджені у книзі Ю. В. Козаченка та ін. [2] у загальному випадку. Однак виникають додаткові труднощі при побудові моделей конкретного прочесу, такі як, наприклад, вибір відповідного базису в $L_{2}(\mathbb{R})$. У чій статті побудовані моделі, які наближають гауссовий прочес зі стійкою кореляиійною фуниіею $\rho_{\alpha}(h)=E X_{\alpha}(t+h) X_{\alpha}(t)=B^{2} \exp \left\{-d|h|^{\alpha}\right\}, \alpha>0, d>0$ з параметром $\alpha=2$, що е центрованим стачіонарним прочесом із заданою надійністю та точністю в просторі $L_{p}([0, T])$. А також знайдено швидкості збіжності моделей, сбормульовано відповідні теореми. Розглянуто способи зображсння та основні властивості прочесу зі стійкою корелячійною фунцією $\rho_{2}(h)=B^{2} \exp \left\{-d|h|^{2}\right\}, d>0$. У якості базису в просторі $L_{2}(\mathbb{T})$ використовуютвся функиї Ерміта.

Ключові слова: кореляиійна фуниія, моделювання, модель прочесу, точність, надійність.

In this paper, the representation of random processes in the form of random series with uncorrelated members obtained in the work by Yu. V. Kozachenko, I.V. Rozora, E.V. Turchina (2007) [1]. Similar constructions were studied in the book by Yu. V. Kozachenko and others. [2] in the general case. However, there are additional difficulties in construction of models of specific process, such as, for example, selection of the appropriate basis in $L_{2}(\mathbb{R})$. In this paper, models are constructed that approximate the Gaussian process with a stable correlation function $\rho_{\alpha}(h)=E X_{\alpha}(t+h) X_{\alpha}(t)=$ $B^{2} \exp \left\{-d|h|^{\alpha}\right\}, \alpha>0, d>0$ with parameter $\alpha=2$, which is a centered stationary process with a given reliability and accuracy in the space $L_{p}([0, T])$. And also the rates of convergence of the models are found, the corresponding theorems are formulated. Methods of representation and main properties of the process with a stable correlation function $\rho_{2}(h)=B^{2} \exp \left\{-d|h|^{2}\right\}, d>0$ are considered. As a basis in the space $L_{2}(\mathbb{T})$ Hermitian functions are used.

Key Words: correlation function, simulation, model of the process, accuracy, reliability.

Статтю представила д.ф.-м.н. Розора I.В.

1 Гауссовий процес зі стійкою кореляційною фунцією

$$
\rho_{2}(h)=B^{2} \exp \left\{-d|h|^{2}\right\}, d>0
$$

Означення 1.1. [3] Стаціонарний випадковий процес $X=\{X(t), t \in[0, T]\}$ називається гауссовим процесом зі стійкою кореляційною фунцією з параметром $\alpha=2$, якщо $E X(t)=0$ та

$$
E(X(t+h) \overline{X(t)})=\sigma^{2} \cdot \exp \left\{-a h^{2}\right\},
$$

де $\sigma^{2}>0, a>0$ - деякі константи.

Щоб отримати зображення процесу у вигляді ряду некорельованих членів, потрібна наступна теорема.

Теорема $1.1([1]))$. Нехай $X=\{X(t), t \in$ $[0, T]\}, E X(t)=0$ е випадковим прочесом другого порядку з кореляиійною функиією $R(t, s)=$ $E(X(t) \overline{X(s)})$. Нехай $\left(\Lambda, \beta_{\Lambda}, \mu\right)$ - вимірний простір з $\sigma$-скінченною мірою $\mu$, функція $f_{i}(t, \lambda)$, 
$t \in T, i=1, \ldots, n$ належать до $L_{2}(\Lambda, \mu)$, ma $\left\{g_{k}(\lambda), k \in \mathbb{R}\right\}$ - ортонормований базис в $L_{2}(\Lambda, \mu)$. Нехай кореляиійна функція $R(t, s)$ може бути представлена у формі

$$
R(t, s)=\sum_{i=1}^{n} \int_{\Lambda} f_{i}(t, \lambda) f_{i}(\bar{t}, \lambda) d \mu(\lambda)
$$

Тоді прочес $Z(t)$ може бути зображсенний у вигляді ряду, збіжного у середнъоквадратичному

$$
X(t)=\sum_{i=1}^{n} \sum_{k \in \mathbb{N}} a_{i k}(t) \xi_{i k},
$$

$\partial e$

$$
\begin{gathered}
\left.a_{i k}(t)=\int_{\Lambda} f_{i}(t, \lambda) g_{k} \overline{(} \lambda\right) d \mu(\lambda), \\
E \xi_{i k}=0, E \xi_{i k} \overline{\xi_{j l}}=\delta_{i j} \delta_{k l} .
\end{gathered}
$$

$\delta_{k l}=\left\{\begin{array}{c}0, k \neq l, \\ 1, k=l\end{array}-\right.$ дельта-функція Кронекера.

Означення 1.2. [4] Функції

$$
g_{k}(u)=\frac{H_{k}(u)}{\sqrt{k !}} \cdot \frac{1}{\sqrt[4]{2 \pi}} \exp \left\{-\frac{u^{2}}{4}\right\}
$$

називаються функціями Ерміта, де

$$
H_{k}(u)=(-1)^{k} e^{u^{2} / 2} \frac{d^{n}}{d u^{n}} e^{-u^{2} / 2}
$$

поліноми Ерміта.

[4] Система функцій

$$
g_{k}(u)=\frac{H_{k}(u)}{\sqrt{k !}} \cdot \frac{1}{\sqrt[4]{2 \pi}} \exp \left\{-\frac{u^{2}}{4}\right\}
$$

- це повна ортонормальна система, тобто

$$
\int_{-\infty}^{\infty} g_{k}(u) g_{m}(u)=\left\{\begin{array}{l}
0, k \neq m \\
1, k=m .
\end{array}\right.
$$

Теорема 1.2. Нехай $X(t)=\{X(t), t \in[0, T]\}-$ гауссовий прочес зі стійкою кореляиійною фуниієюо $\rho_{2}(h)=B^{2} \exp \left\{-d|h|^{2}\right\}, d>0$. Тоді процес $X(t)$ може бути зображенний у вигляді ряду, збіжного у середнъоквадратичному

$$
X(t)=\sum_{k=1}^{\infty} a_{k l}(t) \cdot \xi_{k}+\sum_{k=1}^{\infty} a_{k 2}(t) \cdot \eta_{k},
$$

de $E \xi_{k}=E \eta_{k}=0, E \xi_{k} \xi_{l}=\delta_{k l}, E \eta_{k} \eta_{l}=\delta_{k l}$, $E \xi_{k} \eta_{l}=0$,

$$
a_{k 1}(t)=\int_{-\infty}^{\infty} \frac{\sigma}{(4 \pi a)^{1 / 4}} \cdot \cos t u \cdot e^{-\frac{u^{2}}{8 a}} \cdot g_{k}(u) d u,
$$

$$
a_{k 2}(t)=\int_{-\infty}^{\infty} \frac{\sigma}{(4 \pi a)^{1 / 4}} \cdot \sin t u \cdot e^{-\frac{u^{2}}{8 a}} \cdot g_{k}(u) d u .
$$

Доведення. Доведення цієї теореми випливає з теореми 1.1. В нашому випадку маємо:

$$
\begin{aligned}
R(s, t)= & \sigma^{2} \cdot \exp \left\{-a(t-s)^{2}\right\}= \\
= & \sigma^{2} \frac{1}{\sqrt{4 \pi a}} \int_{-\infty}^{\infty} \cos ((t-s) x) \cdot \exp \left\{-\frac{u^{2}}{4 a}\right\} d x= \\
= & \int_{-\infty}^{\infty}\left(\frac{\sigma}{(4 \pi a)^{1 / 4}} \exp \left\{-\frac{u^{2}}{8 a}\right\} \cos t x\right) \times \\
& \times\left(\frac{\sigma}{(4 \pi a)^{1 / 4}} \exp \left\{-\frac{u^{2}}{8 a}\right\} \cos s x\right) d x+ \\
+ & \int_{-\infty}^{\infty}\left(\frac{\sigma}{(4 \pi a)^{1 / 4}} \exp \left\{-\frac{u^{2}}{8 a}\right\} \sin t x\right) \times \\
& \quad \times\left(\frac{\sigma}{(4 \pi a)^{1 / 4}} \exp \left\{-\frac{u^{2}}{8 a}\right\} \sin s x\right) d x .
\end{aligned}
$$

Тобто, в позначеннях теореми $1.2 n=2, a_{k 1}$ i $a_{k 2}$ визначені в теоремі.

Далі припускаємо, що $X(t)$ - процес Гаусса. $\mathrm{y}$ цьому випадку всі випадкові величини $\xi_{k}$ та $\eta_{k} \in$ незалежними.

\section{2 Модель гауссового стаціонарного про- цесу зі стійкою кореляційною фунцією $\rho_{2}(h)=B^{2} \exp \left\{-d|h|^{2}\right\}, d>0$ в просторі $L_{p}([0, T])$}

Означення 2.1. Модель $X_{N}(t)$ наближає процес $X(t)$ з надійністю

$1-\alpha, \alpha>0$ та точністю $\beta>0$ у просторі $L_{p}([0, T]), p \geqslant 1, t \in[0, T]$, якщо

$$
\begin{aligned}
& P\left\{\left\|X(t)-X_{N}(t)\right\|_{p}>\beta\right\}= \\
& \quad=P\left\{\left(\int_{0}^{T}\left|X(t)-X_{N}(t)\right|^{p} d t\right)^{1 / p}>\beta\right\} \leqslant \alpha .
\end{aligned}
$$


Вісник Київсъкого національного університету імені Тараса Шевченка

2020, 3
Лема 2.1. Нехай $X(t)$ - гауссовий процес зі стійкою кореляиійною фуниією $\rho_{2}(h)=$ $B^{2} \exp \left\{-d|h|^{2}\right\}, d>0$. Якщ, $X_{N}(t)-$ модель иього процесу, тоді має місие така оиінка

$$
\left(\sup _{t \in[0, T]} E\left(X(t)-X_{N}(t)\right)^{2}\right)^{1 / 2} \leqslant B_{N},
$$

$\partial e$

$$
\begin{aligned}
B_{N}= & \frac{\sigma K}{(\pi a)^{1 / 4} \sqrt{N+2}} \times \\
& \times\left(\frac{2 \sqrt{2 \pi a}}{a} T^{2}+4(2 a+1) T+\right. \\
& +\frac{2 \pi a}{a}\left(2 a^{2}+3 a+1\right) .
\end{aligned}
$$

Доведення. 3 теореми 1.2 та означення 1.2 випливає, що

$$
E\left(X(t)-X_{N}(t)\right)^{2}=\sum_{k=N+1}^{\infty}\left(a_{k 1}^{2}(t)+a_{k 2}^{2}(t)\right)
$$

де

$$
\begin{aligned}
& a_{k 1}(t)=\int_{-\infty}^{\infty} \frac{\sigma}{(4 \pi a)^{1 / 4}} \cdot \cos t u \cdot e^{-\frac{u^{2}}{8 a}} \cdot g_{k}(u) d u \\
& a_{k 2}(t)=\int_{-\infty}^{\infty} \frac{\sigma}{(4 \pi a)^{1 / 4}} \cdot \sin t u \cdot e^{-\frac{u^{2}}{8 a}} \cdot g_{k}(u) d u
\end{aligned}
$$

$g_{k}(u)=\frac{H_{k}(u)}{\sqrt{k !}} \cdot \frac{1}{\sqrt[4]{2 \pi}} \exp \left\{-\frac{u^{2}}{4}\right\}$ (див. означення 1.1). Потрібно оцінити $\left|a_{k 1}\right|$ та $\left|a_{k 2}\right|$ :

$\left|a_{k 1}(t)\right|=\left|\int_{-\infty}^{\infty} \frac{\sigma}{(4 \pi a)^{1 / 4}} \cdot \cos t u \cdot e^{-\frac{u^{2}}{8 a}} \cdot g_{k}(u) d u\right|$.

Використовуючи інтегрування частинами, властивості поліномів Ерміта, аналогічно до міркувань, проведених в книзі Ю. В. Козаченко та ін. $([2])$, отримуємо для $\left|a_{k 1}(t)\right|$ наступні співвідношеня

$$
\begin{aligned}
\left|a_{k 1(t)}\right|= & \left|\int_{-\infty}^{\infty} \frac{\sigma}{(4 \pi a)^{1 / 4}} \cdot \cos t u \cdot e^{-\frac{u^{2}}{8 a}} \cdot g_{k}(u) d u\right|= \\
= & \mid \int_{-\infty}^{\infty} \frac{\sigma}{(4 \pi a)^{1 / 4}} \cdot \cos t u \cdot e^{-\frac{u^{2}}{8 a} \times} \\
& \times \frac{H_{k}(u)}{\sqrt{k !}} \cdot e^{-\frac{u^{2}}{4}} \cdot \frac{1}{\sqrt[4]{2 \pi}} d u \mid= \\
= & \mid \frac{1}{\sqrt[4]{2 \pi}} \cdot \int_{-\infty}^{\infty} \frac{\sigma}{(4 \pi a)^{1 / 4}} \cdot \cos t u \cdot e^{-\frac{u^{2}}{8 a}} \times \\
& \times \frac{e^{-\frac{u^{2}}{4}}}{\sqrt{k+1} \sqrt{(k+1) !}} \cdot \frac{\partial H_{k+1}(u)}{\partial u} \cdot d u \mid
\end{aligned}
$$

3 нерівності Крамера [5] випливає, що для будьякого $u$ маємо

$\left|g_{k}(u) \leqslant K\right|$, де $K=1,086435$. Таким чином,

$$
\left|\frac{1}{\sqrt[4]{2 \pi}} \cdot \frac{H_{k}(u)}{\sqrt{(k+1) !}} \exp \left\{-\frac{u^{2}}{4}\right\}\right| \leqslant \text { const. }
$$

Тому $e^{-\frac{u^{2}}{8 a}} \cdot \frac{1}{\sqrt[4]{2 \pi}} \cdot \frac{H_{k}(u)}{\sqrt{(k+1) !}} \exp \left\{-\frac{u^{2}}{4}\right\} \rightarrow 0$, коли $u \rightarrow \pm \infty$. Звідси

$$
\begin{aligned}
\left|a_{k 1}(t)\right|= & \\
= & \mid-\frac{1}{\sqrt[4]{2 \pi}} \int_{-\infty}^{\infty} \frac{\partial\left(\frac{\sigma}{(4 \pi a)^{1 / 4}} \cdot \cos t u \cdot e^{-\frac{u^{2}}{8 a}} \cdot \frac{u^{2}}{4}\right)}{\partial u} \times \\
& \times \frac{H_{k+1}(u)}{\sqrt{k+1} \sqrt{(k+1) !}} \mid
\end{aligned}
$$

За допомогою повторного інтегрування частинами, отримуємо

$$
\begin{aligned}
& \left|a_{k 1}(t)\right|=\mid-\frac{1}{\sqrt[4]{2 \pi}} \int_{-\infty}^{\infty} \frac{\partial\left(\frac{\sigma}{(4 \pi a)^{1 / 4}} \cdot \cos t u \cdot e^{-\frac{u^{2}}{8 a}} \times \frac{u^{2}}{4}\right)}{\partial u} \times \\
& \times \frac{H_{k+1}(u)}{\sqrt{k+1} \sqrt{(k+1) !}} \mid= \\
& =\mid-\frac{1}{\sqrt[4]{2 \pi}} \cdot \int_{-\infty}^{\infty} \frac{\partial\left(\frac{\sigma}{(4 \pi a)^{1 / 4}} \cdot \cos t u \cdot e^{-\frac{u^{2}}{8 a}} \cdot \frac{u^{2}}{4}\right)}{\partial u} \times \\
& \times \frac{1}{\sqrt{(k+1)(k+2)} \sqrt{(k+2) !}} \cdot \frac{\partial H_{k+2}(u)}{\partial u} \mid= \\
& =\mid \frac{1}{\sqrt[4]{2 \pi}} \cdot \int_{-\infty}^{\infty} \frac{\partial\left(\frac{\sigma}{(4 \pi a)^{1 / 4}} \cdot \cos t u \cdot e^{-\frac{u^{2}}{8 a} \cdot \frac{u^{2}}{4}}\right)}{\partial u} \times \\
& \times\left.\frac{1}{\sqrt{(k+1)(k+2)} \sqrt{(k+2) !}} \cdot H_{k+1}(u)\right|_{-\infty} ^{\infty}+ \\
& +\frac{1}{\sqrt[4]{2 \pi}} \cdot \int_{-\infty}^{\infty} \frac{\partial\left(\frac{\sigma}{(4 \pi a)^{1 / 4}} \cdot \cos t u \cdot e^{-\frac{u^{2}}{8 a}} \cdot \frac{u^{2}}{4}\right)}{\partial u^{2}} \times \\
& \times \frac{H_{k+2}(u)}{\sqrt{(k+1)(k+2)} \sqrt{(k+2) !}} d u \cdot H_{k+1}(u) \mid= \\
& =\mid \frac{1}{\sqrt[4]{2 \pi}} \cdot \int_{-\infty}^{\infty} \frac{\partial\left(\frac{\sigma}{(4 \pi a)^{1 / 4}} \cdot \cos t u \cdot e^{-\frac{u^{2}}{8 a}} \cdot \frac{u^{2}}{4}\right)}{\partial u^{2}} \times \\
& \times \frac{H_{k+2}(u)}{\sqrt{(k+1)(k+2)} \sqrt{(k+2) !}} d u \cdot H_{k+1}(u) \mid .
\end{aligned}
$$


3 нерівності Крамера [5] ми отримуємо:

$$
\left|a_{k 1}(t)\right| \leqslant \frac{K}{\sqrt{(k+1)(k+2)}}\left|\int_{-\infty}^{\infty} Z_{1}(t, u) d u\right|
$$

де

$$
\begin{aligned}
Z_{1}(t, u)= & \frac{\partial^{2}\left(\frac{\sigma}{(4 \pi a)^{1 / 4}} \cdot \cos t u \cdot e^{-\frac{u^{2}}{8 a}}\right)}{\partial u^{2}}- \\
& -u \frac{\partial\left(\frac{\sigma}{(4 \pi a)^{1 / 4}} \cdot \cos t u \cdot e^{-\frac{u^{2}}{8 a}}\right)}{\partial u}+ \\
& +\frac{u^{2}-2}{4}\left(\frac{\sigma}{(4 \pi a)^{1 / 4}} \cdot \cos t u \cdot e^{-\frac{u^{2}}{8 a}}\right) .
\end{aligned}
$$

Отже

$$
\begin{aligned}
& \left|\int_{-\infty}^{\infty} Z_{1}(t, u) d u\right|= \\
& =\mid \int_{-\infty}^{\infty} e^{-\frac{u^{2}}{8 a}}\left(\frac { 1 } { 1 6 a ^ { 2 } } \operatorname { c o s } t u \cdot \left(u^{2}(2 a+1)^{2}-\right.\right. \\
& \left.\quad-\left(16 a^{2} t^{2}+8 a^{2}+4 a\right)\right) t u \times \\
& \left.\quad \times \sin t u\left(\frac{1}{2 a}+1\right)\right) d u \mid
\end{aligned}
$$

Оцінимо вираз під знаком інтеграла

$$
\begin{aligned}
& \left|\int_{-\infty}^{\infty} Z_{1}(t, u) d u\right|= \\
& =\mid \frac{\sigma}{(4 \pi a)^{1 / 4}} \int_{-\infty}^{\infty} e^{-\frac{u^{2}}{8 a}}\left(\frac{1}{16 a^{2}} \cos t u \times\right. \\
& \quad \times\left(u^{2}(2 a+1)^{2}-\left(16 a^{2} t^{2}+8 a^{2}+4 a\right)\right)+ \\
& \left.\quad+t u \cdot \sin t u\left(\frac{1}{2 a}+1\right)\right) d u \mid \leqslant
\end{aligned}
$$

$$
\begin{aligned}
& \leqslant \int_{-\infty}^{\infty} \mid \frac{\sigma}{(4 \pi a)^{1 / 4}} \cdot e^{-\frac{u^{2}}{8 a}}\left(\frac{1}{16 a^{2}} \cos t u \times\right. \\
& \quad \times\left(u^{2}(2 a+1)^{2}-\left(16 a^{2} t^{2}+8 a^{2}+4 a\right)\right)+ \\
& \left.\quad+t u \cdot \sin t u\left(\frac{1}{2 a}+1\right)\right) \mid d u \leqslant \\
& \leqslant \int_{-\infty}^{\infty} \frac{\sigma}{(4 \pi a)^{1 / 4}} \cdot e^{-\frac{u^{2}}{8 a}}\left(| \frac { 1 } { 1 6 a ^ { 2 } } | | \operatorname { c o s } t u | \cdot \left(\left|u^{2}(2 a+1)\right|+\right.\right. \\
& \left.+\mid-\left(16 a^{2} t^{2}+8 a^{2}+4 a \mid\right)\right)+ \\
& \left.+|t u| \cdot|\sin t u|\left|\frac{1}{2 a}+1\right|\right) d u \leqslant \\
& \leqslant \int_{-\infty}^{\infty} \frac{\sigma}{(4 \pi a)^{1 / 4}} \cdot e^{-\frac{u^{2}}{8 a}}\left(\frac { 1 } { 1 6 a ^ { 2 } } \cdot \left(u^{2}(2 a+1)^{2}+\right.\right. \\
& \left.\left.+\left(16 a^{2} t^{2}+8 a^{2}+4 a\right)\right)+t|u|\left(\frac{1}{2 a}+1\right)\right) d u= \\
& \quad+t\left(\frac{1}{2 a}+1\right) \int_{-\infty}^{\infty} e^{-\frac{u^{2}}{8 a}} \cdot|u| d u . \\
& \quad=\frac{(2 a+1)^{2}}{16 a^{2}} \cdot \frac{\sigma}{(4 \pi a)^{1 / 4}} \int_{-\infty}^{\infty} e^{-\frac{u^{2}}{8 a}} \cdot u^{2} d u+ \\
& +\left(16 a^{2} t^{2}+8 a^{2}+4 a\right) e^{-\frac{u^{2}}{8 a}} d u+
\end{aligned}
$$

Тепер знайдемо значення наступних інтегралів:

$$
\begin{aligned}
& \int_{-\infty}^{\infty} e^{-\frac{u^{2}}{8 a}} d u= \\
& =\frac{1}{\sqrt{2 \pi} \sqrt{4 a}} \int_{-\infty}^{\infty} e^{-\frac{u^{2}}{2} \cdot \frac{1}{4 a}} d u \sqrt{2 \pi} \sqrt{4 a}=2 \sqrt{2 \pi a}, \\
& \int_{-\infty}^{\infty} e^{-\frac{u^{2}}{8 a}} u^{2} d u= \\
& =\frac{1}{\sqrt{2 \pi} \sqrt{4 a}} \int_{-\infty}^{\infty} e^{-\frac{u^{2}}{2}} u^{2} d u \sqrt{2 \pi} \sqrt{4 a}=4 a \sqrt{2 \pi} \sqrt{4 a}= \\
& =8 a \sqrt{2 \pi a}, \\
& \int_{-\infty}^{\infty} e^{-\frac{u^{2}}{8 a}}|u| d u= \\
& =2 \int_{-\infty}^{\infty} e^{-\frac{u^{2}}{2}} u d u=2 \int_{-\infty}^{\infty} d\left(e^{-\frac{u^{2}}{8 a}}\right) \cdot 4 a=8 a .
\end{aligned}
$$

Замінюємо ці значення на початкову нерівність 
Вісник Київсъкого національного університету імені Тараса Шевченка

$$
\begin{aligned}
&\left|\int_{-\infty}^{\infty} Z_{1}(t, u) d u\right| \leqslant \\
& \leqslant \frac{\sigma}{(4 \pi a)^{1 / 4}}\left(\frac{(2 a+1)^{2}}{16 a^{2}} \int_{-\infty}^{\infty} e^{-\frac{u^{2}}{8 a}} \cdot u^{2} d u+\right. \\
& \quad+\frac{\left(16 a^{2} t^{2}+8 a^{2}+4 a\right)}{16 a^{2}} \int_{-\infty}^{\infty} e^{-\frac{u^{2}}{8 a}} d u+ \\
&\left.\quad+t\left(\frac{1}{2 a}+1\right) \int_{-\infty}^{\infty} e^{-\frac{u^{2}}{8 a}}|u| d u\right)= \\
&=\frac{\sigma}{(4 \pi a)^{1 / 4}}\left(\frac{(2 a+1)^{2}}{16 a^{2}} \cdot 8 a \sqrt{2 \pi a}+\right. \\
&+\frac{\left(16 a^{2} t^{2}+8 a^{2}+4 a\right)}{16 a^{2}} \times \\
&\left.\quad \times 2 \sqrt{2 \pi a}+t\left(\frac{1}{2 a}+1\right) 8 a\right)= \\
&=\frac{\sigma}{(4 \pi a)^{1 / 4}}\left(\frac { \sqrt { 2 \pi a } } { 2 a } \left((2 a+1)^{2}+\right.\right.
\end{aligned}
$$

тоді

$$
\begin{aligned}
& \left.\left.+\left(4 a t^{2}+2 a+1\right)\right)+4 t(2 a+1)\right)= \\
= & \frac{\sigma}{(4 \pi a)^{1 / 4}}\left(\frac{\sqrt{2 \pi a}}{2 a}\left(2 a t^{2}+2 a^{2}+3 a+1\right)+\right. \\
& +4 t(2 a+1))
\end{aligned}
$$

$$
\begin{aligned}
\left|a_{k 1}(t)\right| \leqslant & \frac{K}{\sqrt{(k+1)(k+2)}}\left|\int_{-\infty}^{\infty} Z_{1}(t, u) d u\right| \leqslant \\
\leqslant & \frac{K}{\sqrt{(k+1)(k+2)}} \cdot \frac{\sigma}{(4 \pi a)^{1 / 4}} \times \\
& \times\left(\frac{\sqrt{2 \pi a}}{a}\left(2 a t^{2}+2 a^{2}+3 a+1\right)+\right. \\
& +4 t(2 a+1)) .
\end{aligned}
$$

I $з$ того, що $t \in[0, T]$, маємо

$$
\begin{aligned}
& \sup _{t \in[0, T]]}\left|a_{k 1}(t)\right| \leqslant \\
& \leqslant \frac{K}{\sqrt{(k+1)(k+2)}} \cdot \frac{\sigma}{(4 \pi a)^{1 / 4}} \times \\
& \quad \times\left(\frac{\sqrt{2 \pi a}}{a}\left(2 a T^{2}+2 a^{2}+3 a+1\right)+\right. \\
& \quad+4 T(2 a+1)) .
\end{aligned}
$$

Аналогічно, до міркувань проведених вище, для функції

$$
a_{k 2}(t) \leqslant \int_{-\infty}^{\infty} \frac{\sigma}{(4 \pi a)^{1 / 4}} \cdot \sin t u \cdot e^{-\frac{u^{2}}{8 a}} \cdot g_{k}(u) d u
$$

отримаємо таку оцінку:

$$
\begin{aligned}
& \sup _{t \in[0, T]]}\left|a_{k 2}(t)\right| \leqslant \\
& \leqslant \frac{K}{\sqrt{(k+1)(k+2)}} \cdot \frac{\sigma}{(4 \pi a)^{1 / 4}} \times \\
& \quad \times\left(\frac{\sqrt{2 \pi a}}{a}\left(2 a T^{2}+2 a^{2}+3 a+1\right)+4 T(2 a+1)\right) .
\end{aligned}
$$

I нарешті, отримуємо оцінку для $\sup _{t \in[0, T]} E\left(X(t)-X_{N}(t)\right)^{2}$ :

$$
\begin{aligned}
& \sup _{t \in[0, T]} E\left(X(t)-X_{N}(t)\right)^{2} \leqslant \\
& \leqslant \sum_{k=N+1}^{\infty}\left(\frac{K}{\sqrt{(k+1)(k+2)}}\right)^{2} \times \\
& \quad \times 2\left(\frac { \sigma } { ( 4 \pi a ) ^ { 1 / 4 } } \left(\frac{\sqrt{2 \pi a}}{a}\left(2 a T^{2}+2 a^{2}+3 a+1\right)+\right.\right. \\
& \quad+4 T(2 a+1)))^{2}= \\
& \quad \sigma^{2} K^{2} \\
& \quad\left(\frac{2 \sqrt{2 \pi a}}{a} T^{2}+\right. \\
& \left.\quad+4(2 a+1) T+\frac{\sqrt{2 \pi a}}{a}\left(2 a^{2}+3 a+1\right)\right)^{2} .
\end{aligned}
$$

Беремо квадратний корінь з обох сторін нерівності і отримуємо оцінку цієї леми. 
Теорема 2.1. Нехай $X(t)=\{X(t), t \in[0, T]\}$ - гауссовий вимірний випадковий прочес. Тодi для $\varepsilon>C_{p}^{1 / 2}$, де $C=\int_{0}^{T}\left(E(X(t))^{2}\right)^{p / 2} d t<\infty$, має місце нерівність

$$
P\left\{\|X(t)\|_{p}>\varepsilon\right\} \leqslant 2 \exp \left\{-\frac{\varepsilon^{2}}{2 C^{2 / p}}\right\} .
$$

Доведення. Нерівність (16) випливає з наслідку 2.1 з роботи [6].

Лема 2.2. Нехай $X(t)=\{X(t), t \in[0, T]\}$ вимірний гауссовий прочес зі стійкою кореляиійною фунцією $\rho_{2}(h)=B^{2} \exp \left\{-d|h|^{2}\right\}, d>$ 0. Якщо $X_{N}(t)$ - модель ивого прочессу, тодi справедлива наступна очінка

$$
\int_{0}^{T}\left(E\left(X(t)-X_{N}(t)\right)^{2}\right)^{p / 2} d t \leqslant L_{N}^{p / 2}
$$

$\partial e$

$$
\begin{aligned}
L_{N}= & \frac{\sigma^{2} K^{2}}{(\pi a)^{1 / 2}(N+2)}\left(\frac{8 \pi T^{\frac{2(2 p+1)}{p}}}{a(2 p+1)^{2 / p}}+\right. \\
& +\frac{16 \sqrt{2 \pi a}(2 a+1)}{a} \cdot \frac{T^{\frac{3 p+2}{p}}}{\left(\frac{3 p}{2}+1\right)^{2 / p}}+ \\
& +\left(16(2 a+1)^{2}+\frac{8 \pi}{a}\left(2 a^{2}+3 a+1\right)\right) \times \\
& \times \frac{T^{\frac{2(p+1)}{p}}}{(p+1)^{2 / p}}++8(2 a+1) \frac{\sqrt{2 \pi a}}{a} \times \\
& \times\left(2 a^{2}+3 a+1\right) \frac{T^{\frac{p+2}{p}}}{\left(\frac{p}{2}+1\right)^{2 / p}}+ \\
& \left.+\frac{2 \pi}{a}\left(2 a^{2}+3 a+1\right)^{2} T^{2 / p}\right) .
\end{aligned}
$$

Доведення. Застосовуючи оцінки (9) та (14) 3 леми 2.1, отримаємо

$$
\begin{aligned}
& E\left(X(t)-X_{N}(t)\right)^{2}=\sum_{k=N+1}^{\infty}\left(a_{k 1}^{2}(t)+a_{k 2}^{2}(t)\right), \\
& \sum_{k=N+1}^{\infty}\left(a_{k 1}^{2}(t)+a_{k 2}^{2}(t)\right) \leqslant \\
& \leqslant \frac{\sigma^{2} K^{2}}{(\pi a)^{1 / 2}(N+2)}\left(\frac{2 \sqrt{2 \pi a}}{a} t^{2}+\right. \\
& \left.\quad+4(2 a+1) t+\frac{\sqrt{2 \pi a}}{a}\left(2 a^{2}+3 a+1\right)\right)^{2} .
\end{aligned}
$$

Отже,

$$
\begin{aligned}
&\left(\int_{0}^{T}\left(E\left(X(t)-X_{N}(t)\right)^{2}\right)^{p / 2} d t\right)^{2 / p} \leqslant \\
& \leqslant \frac{\sigma^{2} K^{2}}{(\pi a)^{1 / 2}(N+2)}\left(I_{1}+I_{2}+I_{3}+I_{4}+I_{5}\right)= \\
&= \frac{\sigma^{2} K^{2}}{(\pi a)^{1 / 2}(N+2)}\left(\frac{8 \pi T^{\frac{2(2 p+1)}{p}}}{a(2 p+1)^{2 / p}}+\right. \\
&+ \frac{16 \sqrt{2 \pi a}(2 a+1)}{a} \times \\
& \times \frac{T^{\frac{3 p+2}{p}}}{\left(\frac{3 p}{2}+1\right)^{2 / p}}+\left(16(2 a+1)^{2}+\right. \\
&+\left.\frac{8 \pi}{a}\left(2 a^{2}+3 a+1\right)\right) \cdot \frac{T^{\frac{2(p+1)}{p}}}{(p+1)^{2 / p}}+ \\
&+ 8(2 a+1) \frac{\sqrt{2 \pi a} \cdot T^{\frac{p+2}{p}}}{a\left(\frac{p}{2}+1\right)^{2 / p}}\left(2 a^{2}+3 a+1\right)+ \\
&+\left.\frac{2 \pi}{a}\left(2 a^{2}+3 a+1\right)^{2} T^{2 / p}\right) .
\end{aligned}
$$

де

$$
\begin{gathered}
I_{1}=\left(\int_{0}^{T}\left(\frac{8 \pi}{a} t^{4}\right)^{p / 2} d t\right)^{2 / p} \\
I_{2}=\left(\int_{0}^{T}\left(\frac{16 \sqrt{2 \pi a}(2 a+1)}{a} t^{3}\right)^{p / 2} d t\right)^{2 / p}, \\
I_{3}=\left(\int_{0}^{T}\left(\left(16(2 a+1)^{2}+\frac{8 \pi}{a}\left(2 a^{2}+3 a+1\right)\right) t^{2}\right)^{p / 2} d t\right)^{2 / p}, \\
I_{4}=\left(\int_{0}^{T}\left(8(2 a+1) \frac{\sqrt{2 \pi a}}{a}\left(2 a^{2}+3 a+1\right) t\right)^{p / 2} d t\right)^{2 / p}, \\
\left.I_{5}=\left(\int_{0}^{T}\left(\frac{2 \pi}{a}\left(2 a^{2}+3 a+1\right)^{2}\right)^{p / 2} d t\right)^{2 / p}\right) .
\end{gathered}
$$

Теорема 2.2. Нехай $X(t)=\left\{X_{N}(t), t \in[0, T]\right\}$ - вимірний гауссовий прочес зі стійкою корелячійною фуниією $\rho_{2}(h)=B^{2} \exp \left\{-d|h|^{2}\right\}$, $d>0$. Модель $X_{N}(t)$ наближае прочес $X(t)$ y просторі $L_{p}([0, T])$ з точністю $\beta$ і надійністю $1-\alpha, 0<\alpha<1$, якщо $N>0$ таке, що

$$
L_{N} \leqslant \frac{\beta^{2}}{p}
$$


$m a$

$$
L_{N} \leqslant \frac{\beta^{2}}{(2(\ln 2))}
$$

де $L_{N}$ визначаеться формулою (18).

Доведення. 3 нерівності (16) і леми 2.2, якщо $\varepsilon>L_{N}^{1 / p} p^{1 / 2}$ отримаємо

$$
P\left\{\left|X(t)-X_{N}(t)\right|_{p}>\varepsilon\right\} \leqslant 2 \exp \left\{-\frac{\varepsilon^{2}}{2 L_{N}}\right\} .
$$

Якщо в (21) замість $\varepsilon$ покласти $\beta$, тоді умови теореми виконуються, якщо

$$
\beta \geqslant L_{N}^{p / 2} p^{1 / 2}
$$

\section{Список використаних джерел}

1. Kozachenko Yu.V. On expansion of random process in series / Yu.V. Kozachenko, I.V. Rozora, Ye. V. Turchyn. // Random Operators and Stohastic Equations. - 2007. - v. 15(1). - P. 15-35.

2. Козаченко Ю.В. Квазібанахові простори випадкових величин / Ю.В. Козаченко, Ю.Ю. Млавець, О.М. Моклячук - Ужгород: Карпати, 2015. - 212 с.

3. Uhlenbeck G.E. On the theory of Brownian motion / G.E. Uhlenbeck, L.S. Ornstein. // Phys. Rev. - 1930. - v. 36 P. 832-841.

4. Kampe de Feriet J. Fonctions de la Physique Mathematique / Kampe de Feriet J. - Paris: Paris Editions du CNRS, 1957. - 99 p.

5. Erdelyi A. Higher Transcendental Functions A. Erdelyi. - New-York: McGraw-Hill, 1953. Vol. II - $414 \mathrm{p}$.

6. Kamenschikova $O$. On an expansion of random processes in the space $L_{p}(T) / \mathrm{O}$. Kamenschikova, Y. Kozachenko. // Theory Probab. And Math. Statist. - 2009. - v.79. -P. 83-88.

тобто, якщо виконуються умови (19) і (20), тоді має місце теорема.

\section{3 Висновки}

У данній роботі побудовано моделі, які наближають гауссовий процес зі стійкою кореляційною фунцією $\rho_{2}(h)=B^{2} \exp \left\{-d|h|^{2}\right\}, d>0$ із заданою надійністю $1-\alpha, 0<\alpha<1$ та точністю $\beta>0$ в просторі $L_{p}([0, T]), p \geqslant 1$.

Автор висловлює глибоку подяку своєму керівнику і наставнику Юрію Васильовичу Козаченко. 3 пам'яттю у серці...

\section{References}

1. KOZACHENKO, YU.V., ROZORA, I.V., TURCHYN, YE. V. (2007) On expansion of random process in series. Random Operators and Stohastic Equations. v. 15(1). P. 15-35.

2. KOZACHENKO, YU.V., MLAVETS, YU. YU., MOKLIACHUK, O.M. (2015) Kvazibanakhovi prostory vypadkovykh velychyn. Uzhhorod: Karpaty.

3. UHLENBECK, G.E., ORNSTEIN, L.S. (1930) On the theory of Brownian motion Phys. Rev. v. 36 P. 832-841.

4. KAMPE DE FERIET J. (1957) Fonctions de la Physique Mathematique. Paris: Paris Editions du CNRS.

5. ERDELYI, A. (1953) Higher Transcendental Functions. New-York: McGraw-Hill. Vol. II.

6. KAMENSCHIKOVA, O., KOZACHENKO, Y. (2009) On an expansion of random processes in the space $L_{p}(T)$. Theory Probab. And Math. Statist.. v.79. P. 83-88.

Надійшла до редколегії 01.11.2020 